Gynäkologe 2019· 52:937-944

https://doi.org/10.1007/s00129-019-04536-8

Online publiziert: 7. November 2019

๑ c Der/die Autor(en) 2019

\title{
Redaktion
}

T. Fehm, Düsseldorf

L. Kiesel, Münster

R. Kimmig, Essen

N. Maass, Kiel

\begin{abstract}
Mit der ab 2020 gültigen Krebsfrüherkennungsrichtlinie gelten im deutschen ZervixkarzinomScreening erstmals Algorithmen zur Abklärung auffälliger Befunde. Bisher besteht lediglich Konsens hinsichtlich der Therapiebedürftigkeit einer CIN3 (zervikale intraepitheliale Neoplasie). Das Management bei Zellbildern der Gruppe IIID ebenso wie bei den Gruppen II und III ist durchaus unterschiedlich mit Kontrollzytologie, HPV(humane Papillomviren)-Test, Immunzytochemie und Kolposkopie. Für ein risikoadaptiertes Procedere mit Vermeidung von Überdiagnostik bei Gewährleistung der nötigen Sicherheit für die Patientinnen sollte das Risiko der einzelnen zytologischen Befunde bekannt sein.
\end{abstract}

In Deutschland wurde die Münchner Nomenklatur (MN) für die Zervixzytologie 2014 aktualisiert (MN III; [8]). Unter Beibehaltung der bewährten Gruppenstruktur wurden epithelspezifische Subgruppen eingeführt, welche die Befundtransparenz verbessern und eine Übertragbarkeit ins Bethesda-System (TBS; [25]) ermöglichen. Der wesentliche Unterschied zum TBS ist die dreistufige Graduierung der plattenepithelialen Dysplasiebefunde.

Um bei Patientinnen mit den Befundgruppen II-p, IIID1, III-p und IIID2 das Risiko für CIN2+/CIN3+ beurteilen zu können, haben wir eine Verlaufsbeobachtung durchgeführt. Aufgrund der Patientinnenzahl und der Beobachtungs-

Katrin Marquardt ${ }^{1} \cdot$ Peter Ziemke ${ }^{1} \cdot$ Konrad Neumann $^{2} \cdot$ Wolfgang Kühn ${ }^{3}$

${ }^{1}$ Praxis für Pathologie, Schwerin, Deutschland

${ }^{2}$ Institut für Biometrie und klinische Epidemiologie, Charité Berlin, Berlin, Deutschland

${ }^{3}$ Institut für Zytologie, Reutlingen, Deutschland

\section{Risikobewertung von Zytologiebefunden im Zervixkarzinom-Screening}

dauer erlauben die hier vorgestellten $\mathrm{Da}$ ten Risikobewertungen, welche die statistische Wahrscheinlichkeit von Progression bzw. Regression zytomorphologischer Befunde abbilden.

\section{Material und Methoden}

\section{Erfassung}

Für den Zeitraum vom 02. Januar 2014 (Einführung der MN III) bis 31. Dezember 2016 wurden retrospektiv in einer Praxis für Pathologie (jährlich durchschnittlich 105.000 zytologisch untersuchte Frauen; KM, Schwerin) konsekutiv alle Patientinnen mit plattenepithelialen Befunden der Gruppen II-p, IIID1, III-p, IIID2, IVa-p, IVb-p und V-p $(n=4321)$ anonymisiert erfasst. In die Auswertung wurden alle Frauen mit Erstbefunden, d.h. mit erstmalig auftretenden zytologischen Befunden ohne vorhergehende gleich- oder höhergradige Befunde, aufgenommen ( $\bullet$ Abb. 1). Hysterektomierte Patientinnen wurden ausgeschlossen. Die Verlaufsbeobachtung schloss alle zytologischen Folgebefunde sowie alle histologischen Befunde von den für die abklärenden Einrichtungen zuständigen Pathologen bis 30. Juni 2019 ein. Für die in die Analyse einbezogenen Erstbefunde resultiert ein Beobachtungsumfang von 7346 Personenjahren (2,2 Jahre/Frau). Die Daten wurden in MS Excel in Dreimonatsspalten dokumentiert.

\section{Definitionen der Endpunkte}

Positiver Endpunkt. Die anlässlich einer Biopsie am Beobachtungsende oder der Therapie (Exzision, Laservaporisation) histologisch gestellte Diagnose einer CIN2, CIN3 oder eines Karzinoms.

Negativer Endpunkt. Bei Biopsie am Beobachtungsende oder der Therapie (Exzision, Laservaporisation) histologisch CIN1 oder kein Nachweis von CIN/ Karzinom. Patientinnen ohne Biopsie oder Therapie wurden nach mindestens 2 negativen Abstrichbefunden innerhalb von mindestens 12 Monaten ebenfalls als "negativ“ gewertet.

Zytologisch negativ waren 171 Frauen, ohne die Kriterien für einen negativen Endpunkt zu erfüllen. Weitere 110 Frauen hatten am Beobachtungsende persistierend, rekurrierend oder de novo positive zytologische Befunde (IIID1, III-p, IIID2) und damit keinen auswertbaren Endpunkt. Für 276 Frauen $(7,5 \%)$ mit Erstbefunden konnten keine Folgebefunde ermittelt werden.

\section{Statistische Auswertung}

Für jede Gruppe von Befunden wurde die Zeit-Ereignis-Analyse für die Endpunkte CIN2+ (CIN2, CIN3 und Karzinom) und CIN3+ (CIN3 und Karzinom) anhand von Kaplan-Meier-Schätzungen im Untersuchungszeitraum (maximal $60 \mathrm{Mo}-$ nate) durchgeführt. Die anonymisierten Patientendaten wurden mit IBM SPSS Statistics Version 24 ausgewertet. Weiterhin untersucht wurden der Einfluss 


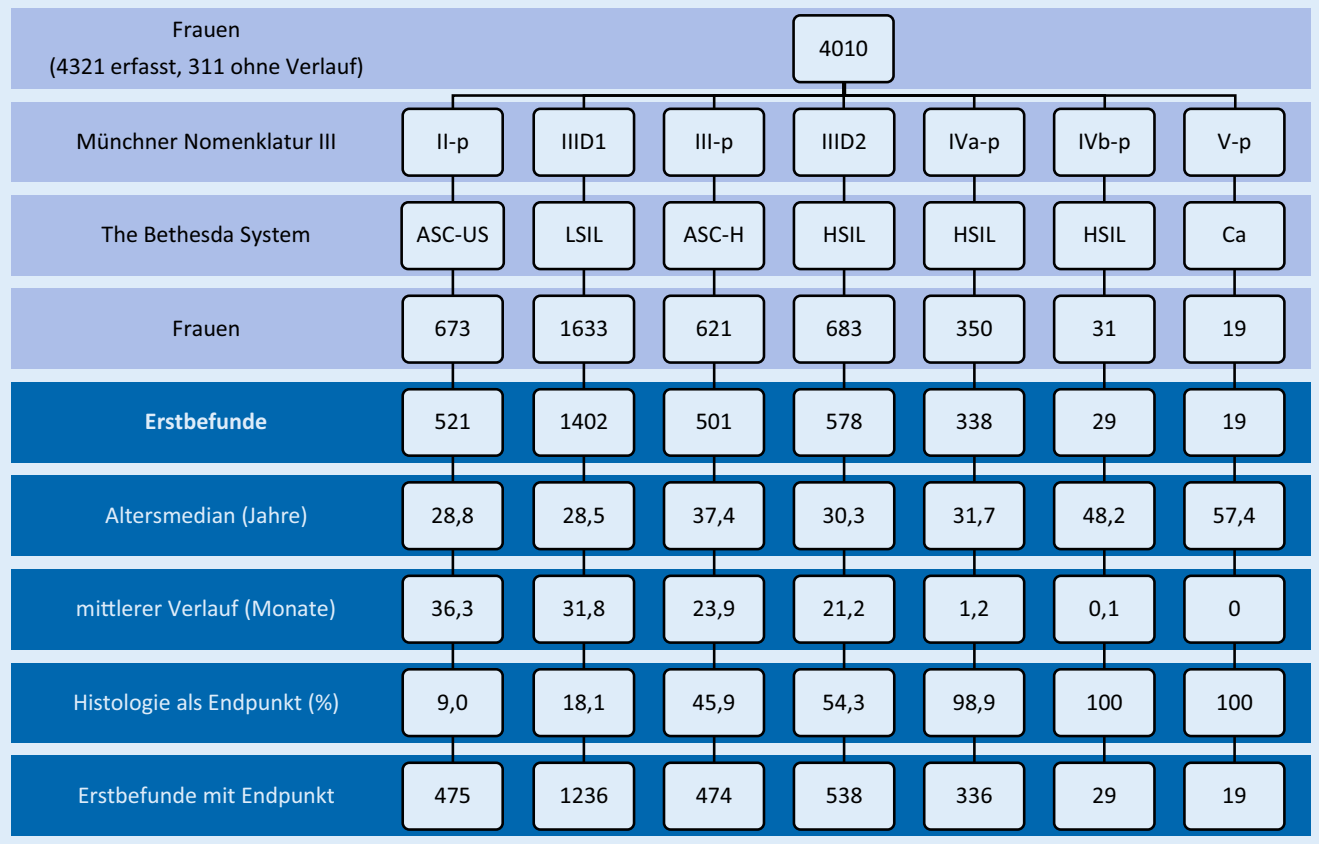

Abb. $1<$ Verteilung der erfassten zytologischen Befunde und Basisdaten erstmaliger zytologischer Befunde, Bethesda-System und Münchner Nomenklatur III. LSIL „low-grade squamous intraepithelial lesion", CIN "cervical intraepithelial neoplasia“" ASC-US "atypical squamous cells of undetermined significance ", ASC-H „atypical squamous cells of undetermined significance cannot exclude HSIL", HSIL "highgrade squamous intraepithelial lesion", Ca Carcinoma

des Lebensalters bei Erfassung und die Auswirkung von Persistenz derselben Befundgruppe auf das Risiko. Diese Berechnungen wurden in MS Excel bzw. online (http.//vassarstats.net) vorgenommen. Signifikanzbestimmungen erfolgten mittels $\chi^{2}$-Test nach Pearson bzw. mittels Fisher-Test. Das Signifikanzniveau betrug 0,05. Alle statistischen Tests waren zweiseitig.

\section{Ergebnisse}

Die Kaplan-Meier-Kurven (• Abb. 2 und 3) zeigen die Risiken für CIN2+ bzw. CIN3+ über einen Zeitraum bis maximal 60 Monate der einzelnen zytologischen Gruppen.

\section{Lebensalter}

Das Risiko für einen Endpunkt CIN2+ und CIN3+ für Patientinnen $<$ bzw. $\geq 35$ Jahre ist für Erstbefunde der einzelnen Befundgruppen in $\bullet$ Tab. 1 dargestellt. Der Vergleich der Altersmittelwerte bei einem positiven Endpunkt CIN2+ und bei Verläufen mit negativem Endpunkt $<$ CIN2 zeigt für die Gruppen II-p (32,6 vs. 31,8$)$, IIID1 (31,8 vs. $30,9)$ und IIID2 (32,7 vs. 32,5) keinen wesentlichen Unterschied. Dagegen un- terscheiden sich für die Gruppe III-p die Altersmittelwerte von 38,6 bei CIN2+ und von 44,2 bei negativem Endpunkt $<$ CIN2 signifikant. Gleichartige Ergebnisse für diese 4 Befundgruppen finden sich bei Vergleich der Frauen $<$ bzw. $\geq 30$ Jahre (nicht tabellarisch dargestellt).

\section{Befundpersistenz}

Das Risiko bei wiederholten Befunden derselben Gruppe zeigt - Tab. 2. Mehrfachbefunde der Gruppe II-p traten in $6,6 \%$, der Gruppe IIID1 in 34,0\%, der Gruppe III-p in 25,7\% und der Gruppe IIID2 in 48,6\% auf. Nur für die Gruppe IIID1 erfolgte eine getrennte Berechnung für zweimalig und mehr als zweimalig aufgetretenen Befund, für die Gruppen II-p, III-p und IIID2 ist dies wegen der zu geringen Fallzahlen nicht sinnvoll.

Der Unterschied zwischen einmaligem und zwei- bzw. mehrmaligem Zytologiebefund hinsichtlich des Risikos für CIN2+ und CIN3+ ist jeweils signifikant.

\section{Karzinome}

Bei den Frauen mit Gruppe II-p ist nach zytologischer Progression (IIID1, IVa-p) nach 29 Monaten ein Karzinom (pT1a1) aufgetreten. Eine gravide $\mathrm{Pa}$ - tientin mit erstmaliger Gruppe IIID1 hatte nach 12 Monaten und zytologischer Progression (IVa-p) ein mikroinvasives Plattenepithelkarzinom. Bei 6 Frauen mit Erstbefund Gruppe IIIp wurde ein Plattenepithelkarzinom diagnostiziert, 2 davon im Stadium pTla1. Nach einer ersten Gruppe IIID2 wurden 4 Plattenepithelkarzinome, davon 3 im Stadium pT1a1 gefunden, nur einmal ohne zytologische Progression.

\section{Diskussion}

Die in Deutschland seit 2014 gültige MN III für die Zervixzytologie [8] führt das Prinzip der risikoorientierten Gruppenstruktur fort, wobei Subgruppen Kompatibilität zum TBS schaffen [25]. Im Gegensatz zum TBS werden Befunde plattenepithelialer Dysplasien dreigliedrig graduiert, um Überdiagnostik und Übertherapie vor allem junger Frauen mit CIN2 zu vermeiden. Die durch die WHO (World Health Organization) seit 2014 auch für die histologischen Befunde eingeführte zweistufige Differenzierung LSIL/HSIL („low-/highgrade squamous intraepithelial lesion“) wird einerseits mit dem bereits bei CIN2 vorhandenen „signifikanten“ Risiko für ein Karzinom begründet, andererseits 
mit der großen Interobserver-Variabilität bei CIN2 [5, 27]. Die histologischen Diagnosen unserer Patientinnen weisen, wie in Deutschland üblich, zusätzlich zu „HSIL“ stets „CIN2“ oder „CIN3“ aus. Die zytologisch-histologische Korrelation lässt sich dadurch exakt darstellen.

Beim Zellbild einer CIN3 (Gruppe IVa-p, HSIL/,severe dysplasia/carcinoma in situ“) und korrelierendem kolposkopischem Befund besteht Konsens über die Indikation zur Therapie mittels LLETZ („large loop excision of the transformation zone“; $[15,22$, 23]). Demgegenüber ist das klinische Management mit Einsatz von Kontrollzytologie, HPV-Test, p16-/Ki-67Immunzytochemie und Kolposkopie bei den Gruppen II, III und IIID bzw. bei ASC-US („atypical squamous cells of undetermined significance"), ASC-H (, atypical squamous cells of undetermined significance cannot exclude highgrade squamous intraepithelial lesion“) und LSIL („low-grade squamous intraepithelial lesion") uneinheitlich und wird kontrovers diskutiert. Algorithmen aus anderen Ländern können nicht ohne weiteres auf das deutsche Screening-System übertragen werden. Während für TBS Follow-up-Studien zu ASC-US und LSIL durchgeführt wurden, liegen für MN III nur wenige Verlaufsbeobachtungen vor [20]. Unsere Analysen mit zytologischen und histologischen Verlaufsdaten für die Gruppen II-p, IIID1, III-p und IIID2 stellen erste Längsschnittuntersuchungen zur MN III an einem großen Kollektiv mit langer Beobachtungsdauer von bis zu 60 Monaten dar. Laborstruktur und Befunderhebung unterliegen dem in Deutschland seit 1971 praktizierten opportunistischen Screening-Modell mit dem Angebot einer jährlichen zytologischen Untersuchung. Mit diesem Konzept und den Abklärungsmodalitäten bei auffälliger Zytologie konnte die Inzidenz des Zervixkarzinoms um mehr als $70 \%$ gesenkt werden $[10,26]$. Bei der Restinzidenz des Zervixkarzinoms unter Screening-Teilnehmerinnen handelt es sich überwiegend um prognostisch günstige Karzinome [4, 19].

Die Kaplan-Meier-Schätzung zeigt, dass eine zytologische Differenzierung von 3 Schweregraden bei sig-

Gynäkologe 2019·52:937-944 https://doi.org/10.1007/s00129-019-04536-8

(c) Der/die Autor(en) 2019

K. Marquardt · P. Ziemke · K. Neumann · W. Kühn

\section{Risikobewertung von Zytologiebefunden im Zervixkarzinom- Screening}

\section{Zusammenfassung}

Zielstellung. Das CIN2+-/CIN3+(zervikale intraepitheliale Neoplasie)-Risiko plattenepithelialer zytologischer Befunde (Gruppe II-p, IIID1, III-p, IIID2 der Münchner Nomenklatur [MN] III) soll bewertet und mit Empfehlungen für das Patienten-Management korreliert werden.

Material und Methoden. Alle 4321 Frauen mit einer Gruppe II-p, IIID1, III-p, IIID2, IVa-p, IVb-p und V-p von Januar 2014 bis Dezember 2016 wurden in einer Praxis für Pathologie erfasst. Zytologische Vorbefunde, alle zytologischen und histologischen Folgebefunde bis Juli 2019 wurden dokumentiert. Für die Erstbefunde wurde pro Befundgruppe mittels Kaplan-Meier-Schätzung das Risiko für CIN2+/CIN3+ ermittelt. Das Risiko bei persistierenden Befunden und der Einfluss des Alters wurden untersucht.

Ergebnisse. Für die Erstbefunde der Gruppen II-p, IIID1, III-p und IIID2 beträgt das Risiko für CIN2+ nach 12, 24 und 60 Monaten $1,0 / 3,3 / 11,8 ; 3,1 / 9,4 / 22,7 ; 33,4 / 38,4 / 55,5$ bzw. 35,1/46,2/64,4 (\%). Das Risiko für
CIN3+ beträgt für die gleichen Zeiträume $0,6 / 2,7 / 9,7 ; 1,9 / 5,2 / 14,3 ; 28,8 / 32,4 / 44,0$ bzw. 28,5/36,7/52,4 (\%). Bei persistierenden Befunden derselben Gruppe steigt das Risiko für CIN2+ und CIN3+ signifikant vom ersten zum zweiten II-p-, IIID1-, III-p- und IIID2-Befund. Bei den Gruppen II-p, IIID1 und IIID2 finden sich keine signifikanten Altersunterschiede, bei III-p-Befunden ist das Risiko für Frauen $<30$ und $<35$ signifikant größer als für ältere Frauen.

Schlussfolgerung. Die MN III mit 3-gliedriger Dysplasiegraduierung klassifiziert die zytologischen Befunde risikoorientiert. Ihre Befundgruppen eignen sich als Basis für ein risikoadaptiertes Management, um insbesondere Überdiagnostik und -therapie zu vermeiden.

\section{Schlüsselwörter}

Zervixkarzinom-Screening · Zervixzytologie . Zervikale intraepitheliale Neoplasie . Münchner Nomenklatur III · RisikoAssessment

\section{Risk assessment of cytology findings in cervical cancer screening}

Abstract

Aim. The risk of progression of squamous cytological findings (Group II-p, IIID1, III-p, IIID2 of the Munich Nomenclature III) is evaluated and correlated with recommendations for patient management.

Methods. Between January 2014 and December 2016, 4321 women were diagnosed as group II-p, IIID1, III-p, IIID2, IVa-p, IVb-p and V-p and represent the study population. Follow-up information on all subsequent cytologic and histologic findings were collected up to July 2019. For the initial cytologic diagnosis in each category of findings, the cumulative risk of $\mathrm{CIN} 2+$ or CIN3+ was determined by Kaplan-Meier estimates. Furthermore, the risk for persistent pathologic findings was calculated and the influence of age analyzed.

Results. The cumulative risk of $\mathrm{CIN} 2+$ for initial findings II-p, IIID1, III-p and IIID2 is calculated after 12,24 and 60 months as (\%) $1.0 / 3.3 / 11.8 ; 3.1 / 9.4 / 22.7 ; 33.4 / 38.4 / 55.5$ and $35.1 / 46.2 / 64.4$, respectively. The cumulative risk of $\mathrm{CIN} 3+$ is determined as (\%) $0.6 / 2.7 / 9.7 ; 1.9 / 5.2 / 14.3 ; 28.8 / 32.4 / 44.0$ and $28.5 / 36.7 / 52.4$, respectively. For persistent cytologic diagnoses II-p, IIID1, III-p and IIID2 the risk of both CIN2+ and CIN3+ increases significantly after the second result. Risk of CIN2+ and CIN3+ for group III-p is significantly higher for women both $<35$ years and $<30$ years compared with women of higher age. There are no significant agedependent differences for findings II-p, IIID1 und IIID2.

Conclusions. The Munich Nomenclature III classifies cytologic findings according to risk. The diagnostic groups of the Munich Nomenclature III are suitable for risk-adapted clinical management, especially to avoid overdiagnosis and overtreatment.

\section{Keywords}

Cervical cancer screening - Cervical cytology . Cervical intraepithelial neoplasia · Munich nomenclature III · Risk assessment 


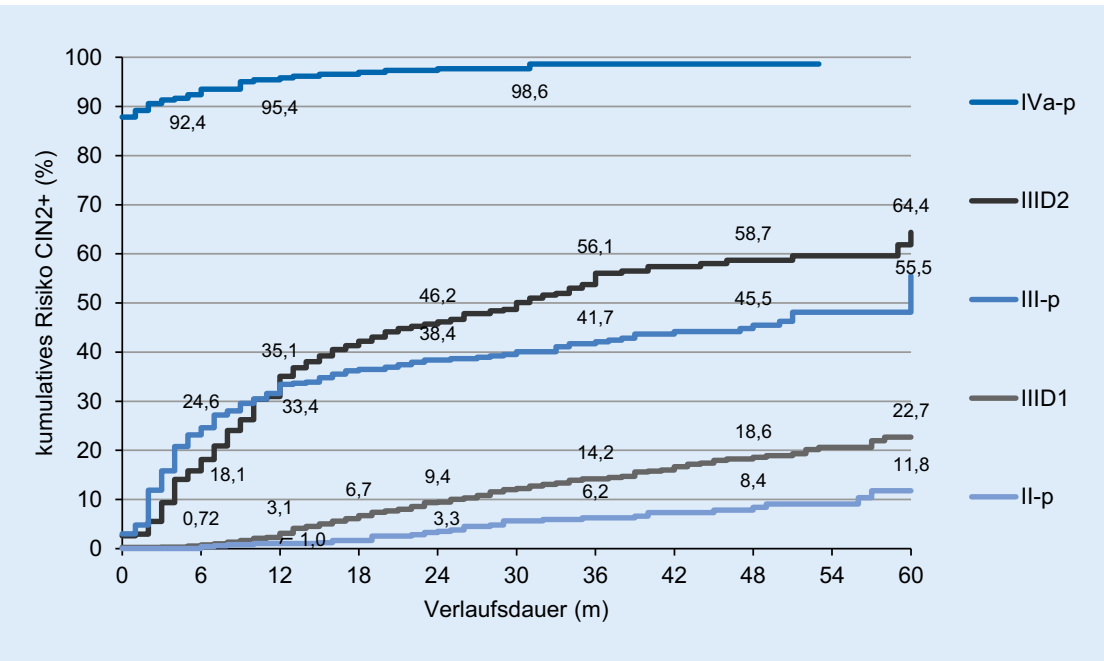

Abb. 2 ム Kumulatives Risiko für CIN2+ (zervikale intraepitheliale Neoplasie) erstmaliger plattenepithelialer Zytologiebefunde der Münchner Nomenklatur III im zeitlichen Verlauf (Kaplan-Meier-Schätzung)

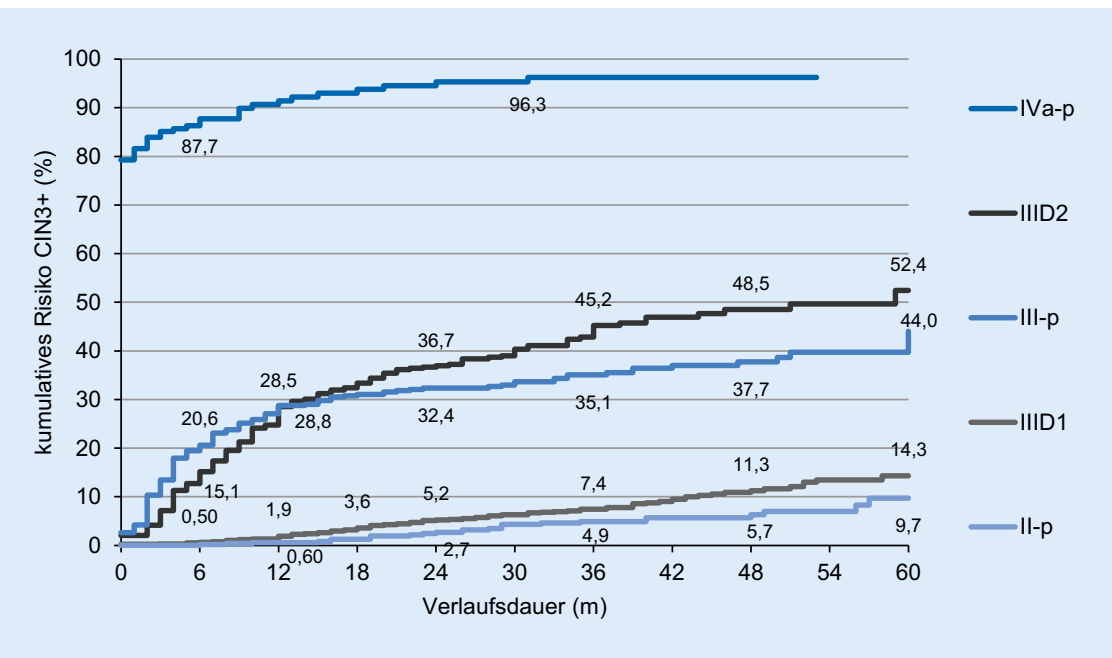

Abb. 3 ॥ Kumulatives Risiko für CIN3 + (zervikale intraepitheliale Neoplasie) erstmaliger plattenepithelialer Zytologiebefunde der Münchner Nomenklatur III im zeitlichen Verlauf (Kaplan-Meier-Schätzung)

nifikant unterschiedlichem Risiko für CIN2+/CIN3+ möglich ist und eine sichere Überwachung der Läsion unter Vermeidung unnötiger diagnostischer Maßnahmen erlaubt.

Die Gruppe IIID2 („HSIL/moderate dysplasia“) wird bisher zytologisch sowie durch kolposkopisch geführte Biopsie überwacht und ggf. durch Exzision therapiert $[9,15,16,24]$. Die Empfehlung, bereits nach erstmaliger Gruppe IIID2 zwingend eine Kolposkopie durchzuführen [3, 7], lässt sich aus unseren Berechnungen nach dem Kaplan-MeierVerfahren nicht nachvollziehen. Das Ri- siko für eine CIN3+ ist derart gering, dass eine kolposkopische Abklärung erst bei Persistenz/Progression in der Kontrollzytologie nach 3 Monaten notwendig wird. Von 216 Patientinnen mit einer CIN3+-Diagnose nach Gruppe IIID2 erhielten im Verlauf 200 Frauen (93\%) Folgebefunde der Gruppe III-p, IIID2 oder IVa-p, woraufhin die Abklärung erfolgte. Eine unmittelbare Kolposkopie führt zweifellos zur früheren Entdeckung einer bereits unterliegenden CIN3. Jedoch zeigen unsere Daten, dass bei erstmaliger Gruppe IIID2 in $62 \%$ am Verlaufsende keine CIN3 vorhanden ist.
Für die Gruppe IIID1 (LSIL) sind die Risikowerte der Erstbefunde höher als Ergebnisse aus der Literatur (nach 5 Jahren für CIN2+ $16 \%$, CIN3 $+5,6 \%$ ), wobei Unterschiede im Studiendesign zu berücksichtigen sind $[1,12]$. Hinter Befunden der Gruppe IIID1 können sich mittel- und hochgradige CIN verbergen bzw. im Verlauf entwickeln. Das Risiko, dass ein Karzinom vorliegt oder in kürzerer Frist entstehen könnte, ist hingegen auch nach unseren Daten mit einem Beobachtungszeitraum von bis zu 5 Jahren äußerst gering. In einer Follow-up-Studie mit über 38.000 Frauen mit LSIL wurden innerhalb von 6 Monaten 6000 Befunde histologisch ohne Karzinomdiagnose abgeklärt [29]. Dass LSIL-Patientinnen ein Niedrigrisikokollektiv für ein Zervixkarzinom darstellen, hatte zudem 2016 eine schwedische Follow-up-Studie mit Beobachtungszeiten von mehr als 10 Jahren anhand großer Zahlen nachgewiesen [28]: In nur 0,5\% fanden sich Karzinome. In einer prospektiven Follow-up-Studie an HPV-positiven Frauen mit LSIL und „unauffälliger" Kolposkopie betrug das Risiko für eine CIN3+ bei einem 3-Jahresintervall ebenfalls nur 4,4\% [14].

\section{》) In der ASCUS-LSIL Triage Study betrug die high-risk HPV- Positivität bei LSIL mehr als $80 \%$}

Bei unseren Patientinnen war das Risiko für CIN3+ bei einmaligem IIID1-Befund sehr gering und lag auch nach mehr als zweimaliger Befundwiederholung für $\mathrm{CIN} 3+$ unter $10 \%$. Orientiert man sich an US-amerikanischen und deutschen Risikoschwellenwerten für die Kolposkopie von $10 \%$ für eine therapiebedürftige Läsion (in Schweden 20\%; [3]), so kann die Gruppe IIID1 zunächst ausschließlich zytologisch kontrolliert werden. Der HPV-Status ist für die Frauen mit Gruppe IIID1 unserer Analyse nur für $20 \%$ bekannt, zeigt hier aber eine Positivitätsrate von $79 \%$. Nach den Daten der ALTS (ASCUS-LSIL Triage Study) betrug die high-risk HPV-Positivität bei LSIL mehr als $80 \%$ [2]. Eine Kolposkopie erscheint demnach - unabhängig vom HPV-Testergebnis - erst nach Persistenz über 24 Monate notwendig, sie dient dem 
Tab. 1 Altersabhängigkeit des Risikos für CIN2 + und CIN3 + für nicht primär therapiebedürftige Zytologiebefunde der MN III bei Vergleich der Frauen $<35$ und $\geq 35$ Jahre

\begin{tabular}{|c|c|c|c|c|c|c|c|}
\hline \multirow[t]{2}{*}{ Gruppe } & \multirow{2}{*}{$\begin{array}{l}\text { Mittleres } \\
\text { Alter } \\
\text { (Jahre) }\end{array}$} & \multirow{2}{*}{$\begin{array}{l}\text { Alters- } \\
\text { gruppe }\end{array}$} & \multirow{2}{*}{$\begin{array}{l}\text { Anzahl } \\
\text { Frauen }\end{array}$} & \multicolumn{2}{|c|}{ Risiko (\%) } & \multicolumn{2}{|l|}{$p$} \\
\hline & & & & CIN2+ & CIN3+ & CIN2+ & CIN3+ \\
\hline \multirow[t]{2}{*}{$\|-p$} & \multirow[t]{2}{*}{31,8} & $<35$ & 316 & 7,3 & 5,7 & \multirow[t]{2}{*}{0,8875} & \multirow[t]{2}{*}{0,7401} \\
\hline & & $\geq 35$ & 158 & 6,9 & 5,0 & & \\
\hline \multirow[t]{2}{*}{ IIID1 } & \multirow[t]{2}{*}{31,1} & $<35$ & 891 & 15,0 & 8,4 & \multirow[t]{2}{*}{1} & \multirow[t]{2}{*}{0,8625} \\
\hline & & $\geq 35$ & 345 & 15,1 & 8,1 & & \\
\hline \multirow[t]{2}{*}{ III-p } & \multirow[t]{2}{*}{41,8} & $<35$ & 198 & 53,5 & 42,4 & \multirow[t]{2}{*}{0,00013} & \multirow[t]{2}{*}{0,00242} \\
\hline & & $\geq 35$ & 276 & 35,9 & 29,0 & & \\
\hline \multirow[t]{2}{*}{ IIID2 } & \multirow[t]{2}{*}{32,6} & $<35$ & 358 & 55,3 & 42,7 & \multirow[t]{2}{*}{0,1990} & \multirow[t]{2}{*}{0,0843} \\
\hline & & $\geq 35$ & 180 & 49,4 & 35,0 & & \\
\hline
\end{tabular}

CIN zervikale intraepitheliale Neoplasie, MN Münchner Nomenklatur

Tab. 2 Risiko für CIN2+ und CIN3+ bei ein-/mehrmaligem Auftreten ausschließlich derselben Befundgruppe (primär nicht therapiebedürftige Zytologiebefunde der MN III)

\begin{tabular}{|c|c|c|c|c|c|c|c|c|c|c|c|}
\hline \multirow[t]{2}{*}{ Gruppe } & \multirow{2}{*}{$\begin{array}{l}\text { Anzahl } \\
\text { Frauen }\end{array}$} & \multirow{2}{*}{$\begin{array}{l}\text { Befunde } \\
\text { pro } \\
\text { Frau }\end{array}$} & \multirow{2}{*}{$\begin{array}{l}\text { Mittlerer } \\
\text { Verlauf } \\
\text { (Monate) }\end{array}$} & \multicolumn{4}{|c|}{ Risiko CIN2+ } & \multicolumn{4}{|c|}{ Risiko CIN3+ } \\
\hline & & & & Alle & $1 x$ & $2 x$ & $>2 x$ & Alle & $1 x$ & $2 x$ & $>2 x$ \\
\hline$\|-p$ & 362 & 1,1 & 37,4 & 0,55 & 0,30 & 4,2 & & 0,28 & 0 & 4,2 & \\
\hline IIID1 & 980 & 1,6 & 34,8 & 3,4 & 0,46 & 3,9 & 17,5 & 1,4 & 0,31 & 1,9 & 6,4 \\
\hline III-p & 304 & 1,3 & 28,5 & 24,3 & 8,0 & 71,8 & & 17,8 & 7,1 & 48,7 & \\
\hline IIID2 & 286 & 1,9 & 24,1 & 35,7 & 12,2 & 60,4 & & 23,4 & 8,2 & 39,6 & \\
\hline
\end{tabular}

CIN zervikale intraepitheliale Neoplasie, $M N$ Münchner Nomenklatur

Ausschluss von „major changes“ als Korrelat für eine CIN2+. Diese Schlussfolgerungen stehen im Gegensatz zu den Empfehlungen der deutschen S3-Leitlinie [3] und zu den darauf gründenden Algorithmen der KrebsfrüherkennungsRichtlinie [7]. Eine frühzeitige Kolposkopie trägt lediglich dazu bei, eine mögliche CIN2/3 zu einem früheren Zeitpunkt als bei einer ausschließlich zytologischen Kontrolle zu erkennen, sodass sie bei Frauen mit schlechter Compliance in Betracht kommt.

Bei der Gruppe III-p (ASC-H) mit differenzialdiagnostischer Erwägung einer CIN2/CIN3 bzw. eines Karzinoms zeigt sich eine hohe Interobserver-Variabilität und damit eine größere Varianz hinsichtlich der Häufigkeit in verschiedenen Laboren. In Deutschland wurden für 2015 und 2016 0,11 \% III-p-Fälle berichtet [21], dieser Anteil ist der ASC-HRate aus den USA vergleichbar [6]. Das Wortgutachten des Zytologen mit Nennung der jeweiligen Differenzialdiagnose (CIN2, CIN3 oder Karzinom) ist Voraussetzung für ein angemessenes Procedere. Ein einmaliger III-p-Befund hat ein
CIN3+-Risiko unter $10 \%$, sodass bei Erwägung einer CIN zunächst eine zytologische Kontrolluntersuchung vertretbar ist. Die Risikosteigerung vom ersten zum zweiten III-p-Befund ist so erheblich, dass an dieser Stelle eine kolposkopische Abklärung zwingend erscheint.

\section{I) Das zytologische Gutachten mit Differenzialdiagnose ist Voraussetzung für ein angemessenes Procedere}

Die Gruppe III-p zeigt im Gegensatz zu den Gruppen II-p, IIID1 und IIID2 ein altersabhängig hoch signifikant unterschiedliches Risiko sowohl beim Vergleich der Patientinnen $<$ bzw. $\geq 35$ als auch $<$ bzw. $\geq 30$ Jahre, wobei das Risiko für CIN2+ und CIN3+ bei den jüngeren Frauen signifikant höher ist. Deshalb ist für die Befundgruppen II-p, IIID1 und IIID2 ein aggressiveres Procedere für ältere Frauen aufgrund des Risikos nicht zu begründen, wohingegen eine Gruppe III- p auch bei jüngeren Frauen zuverlässig abzuklären ist.

In Übereinstimmung mit Untersuchungen zur vergleichbaren TBSKategorie ASC-US zeigt die von uns untersuchte Gruppe II-p, bei der differenzialdiagnostisch allenfalls eine CIN1 erwogen wird, ein geringes Risiko für CIN2+/CIN3. Im Vergleich mit Literaturdaten für negative Zytologiebefunde ist dieses Risiko jedoch deutlich erhöht [13], was die Existenz und Anwendung dieser Befundgruppe rechtfertigt. In Deutschland wurden 2015 und 2016 bei 15 Mio. untersuchten Frauen 1,13 bzw. $0,99 \%$ II-p-Fälle registriert [21]. Trotz bekannter hoher Interobserver-Variabilität mit einem $\kappa-M a ß$ von lediglich 0,2 [18] und obwohl die morphologischen Kriterien für ASC-US zwischen den einzelnen Zytologen differieren können, ist der extreme Unterschied zur Häufigkeit von ASC-US in den USA (4,3\%; [6]) allein aus der Morphologie kaum zu erklären. Denkbar ist ein erhöhtes Sicherheitsbedürfnis der TBS-Anwender bei geringen Atypien: Die Anteile von LSIL $(2,5 \%$; [6]) und Gruppe IIID1 $(0,74 \%$; [21]) unterscheiden sich ebenfalls deutlich.

In der vorliegenden Untersuchung weisen Erstbefunde der Gruppe II-p mit einer durchschnittlichen Beobachtungszeit von 36 Monaten ohne nachfolgende zytologische Progression selbst bei Befundpersistenz ein geringes Risiko für CIN2+/CIN3 auf (je eine Frau mit CIN2 bzw. CIN3). Ähnliche Ergebnisse sind in der Literatur angegeben (nach 5 Jahren $\mathrm{CIN} 2+6,9 \%, \mathrm{CIN} 3+2,6 \%$; [11]). Zervixkarzinome kommen in den wenigsten Studien vor. Die deutsche S3Leitlinie und die KrebsfrüherkennungsRichtlinie sehen bei HPV-positiven IIp-Befunden eine Kolposkopie vor [3, 7]. Der HPV-Status wurde nur in $7 \%$ unserer erstmaligen II-p-Fälle erhoben. Das Patienten-Management erfolgte unabhängig davon. Unsere Daten weisen darauf hin, dass sich bei definitionsgemäßer Gruppenzuordnung das Risiko für die Patientin nicht erhöht, wenn auf einen HPV-Test, eine zytologische Kontrolle bereits nach 6 Monaten und/ oder eine Kolposkopie verzichtet wird. In einer Metaanalyse ergab sich in einem 
Vergleich von zytologischen Kontrollen und frühzeitiger Kolposkopie bei ASCUS keine Überlegenheit für eine dieser Methoden [17].

Unsere Schlussfolgerungen für das Procedere bei auffälligen zytologischen Befunden sollten zurückhaltend bewertet werden. Die retrospektiv erfassten zytologischen Befunde sind in der Routine eines einzelnen zytologischen Labors erstellt worden. Eine histologische Abklärung wurde - mit Ausnahme der Gruppen IV und V - primär äußerst selten vorgenommen. Kolposkopie, HPVTestung oder p16-/Ki-67-Immunhistochemie im Verlauf erfolgten nach individueller Befundkonstellation und wurden nicht in die Auswertung einbezogen. Weitere wichtige Risikofaktoren wie Rauchen, Sexualanamnese, Immunsuppression etc. wurden nicht erfasst.

Künftig wird im deutschen organisierten Früherkennungsprogramm bei über 35-jährigen Frauen aus der Ko-Testung zusätzlich zur Zytologie der HPV-Status bekannt sein. Bei Frauen unter 35 soll der HPV-Test, Algorithmen folgend, in die Abklärung einbezogen werden. Die Synopse der Befunde wird das Procedere bestimmen. Gerade deshalb ist es von Interesse, bei der bekannten schlechten Spezifität des HPV-Testes die Risikowerte der zytologischen Befunde zu kennen.

\section{Fazit für die Praxis}

- Die Münchner Nomenklatur III klassifiziert zytologische Befunde risikoabhängig.

- Die Unterscheidung von 3 DysplasieGraden ermöglicht ein differenziertes Patienten-Management.

- Bei Patientinnen mit einem Endpunkt CIN2+ nach Niedrigrisikoerstbefunden II-p und IIID1 traten im Verlauf fast immer höhergradig atypische Zytologie-Befunde auf, sodass ein abwartendes Procedere vorzuziehen ist.

- Für II-p-, IIID1- und IIID2-Befunde bestehen keine Risikounterschiede zwischen Frauen <30/35 und $\geq 30 / 35$ Jahren. Deshalb lässt sich eine altersabhängig unterschiedliche Abklärungsstrategie aufgrund des Risikos nicht begründen.

\section{- Unabhängig von Algorithmen erfor- dert jeder Einzelfall eine ärztliche Bewertung unter Berücksichtigung von zytologischer Anamnese und weiteren möglichen Risikofaktoren.}

\section{Korrespondenzadresse}

\section{Dr. Katrin Marquardt}

Praxis für Pathologie

Güstrower Str. 34, 19055 Schwerin, Deutschland katrin.marquardt.schwerin@gmail.com

\section{Einhaltung ethischer Richtlinien}

Interessenkonflikt. K. Marquardt, P. Ziemke, K. Neumann und W. Kühn geben an, dass kein Interessenkonflikt besteht.

Für diesen Beitrag wurden von den Autoren keine Studien an Menschen oder Tieren durchgeführt. Für die aufgeführten Studien gelten die jeweils dort angegebenen ethischen Richtlinien.

Open Access Dieser Artikel wird unter der Creative Commons Namensnennung 4.0 International Lizenz (http://creativecommons.org/licenses/by/4.0/deed. de) veröffentlicht, welche die Nutzung, Vervielfältigung, Bearbeitung, Verbreitung und Wiedergabe in jeglichem Medium und Format erlaubt, sofern Sie den/die ursprünglichen Autor(en) und die Quelle ordnungsgemäß nennen, einen Link zur Creative Commons Lizenz beifügen und angeben, ob Änderungen vorgenommen wurden.

\section{Literatur}

1. Alanen KW, Elit LM, Molinaro PA, McLachlin CM (1998) Assessment of cytologic follow-up as the recommended management for patients with atypical squamous cells of undetermined significance or low grade squamous intraepithelial lesions. Cancer 84:5-10

2. The ASCUS-LSIL Triage Study (ALTS) Group (2000) Human Papillomavirus testing for triage of women with cytologic evidence of low-grade squamous Intraepithelial lesions: baseline data from a randomized trial. J Natl Cancer Inst 92:397-402

3. AWMF (2017) S3-Leitlinie Prävention des Zervixkarzinoms. http://www.awmf.org/leitlinien/ detail/II/015-027OL.html. Zugegriffen: 3. Juli 2019

4. Castle PE, Kinney WK, Cheung LC, Gage LC, Fetterman B, Poitras NE, Lorey TS, Wentzensen N, Befano B, Schussler J, Katki HA, Schiffman M (2017) Why does cervical cancer occur in a state-of-the-art screening program? Gynecol Oncol 146:546-553

5. Darragh TM, Colgan TJ, Cox JT, Heller DS, Henry MR, Luff RD, McCalmont T, Nayar R, Palewsky JM, Wilkinson EJ, Zaino RJ, Wilbur DC (2012) The lower Anogenital squamous terminology standardization project for HPV-associated lesions: background and consensus recommendations from the college of American pathologists and the American society for Colposcopy and cervical pathology. Arch Pathol Lab Med 136:1266-1297
6. Eversole GM, Moriarty AT, Schwartz MR, Clayton AC Souers R, Fatheree LA, Chmara BA, Tench WD, Henry MR, Wilbur DC (2010) Practises of participants in the college of American pathologists Interlaboratory comparison program in Cervicovaginal cytology, 2006. Arch Pathol Lab Med 134:331-335

7. Gemeinsamer Bundesausschuss (2018) Krebsfrüherkennungsrichtlinie. https://www.g-ba.de/ downloads/39-261-3597/2018-11-22_oKFE-RL_ Zervixkarzinom.pdf.Zugegriffen: 9. Mai 2019

8. Griesser H, Marquardt K, Jordan B, Kühn W, Neis K, Neumann HH, Bollmann R, Pöschel B, Steiner M, Schenck U (2013) Münchner Nomenklatur III. Frauenarzt 54:2-7

9. Griesser H, Marquardt K, Jordan B, Küppers V, Gieseking F, Kühn W (2015) Das Prozedere bei auffälligen Befunden. Kommentar zur Münchner Nomenklatur III. Frauenarzt 56:10-13

10. IARC (2018) Cancer fact sheets. http://globocan. iarc.fr/pages/fact_sheets_cancer.aspx. Zugegriffen: 3. Juli 2019

11. Katki HA, Schiffman M, Castle PE, Fetterman B, Poitras NE, Lorey T, Cheung LC, Raine-Bennett T, Gage JC, Kinney WK (2013) Five-year risk of CIN3+ and cervical cancer for women with HPV testing of ASC-US Pap results. J Low Genit Tract Dis 17(501):36-42

12. Katki HA, Schiffman $M$, Castle PE, Fetterman $B$, Poitras NE, Lorey T, Cheung LC, Raine-Bennett T, Gage JC, Kinney WK (2013) Five-year risks of CIN2+ and CIN3+ for women with HPV-positive and HPVnegative LSIL Pap results. J Low Genit Tract Dis 17(501):43-49

13. Katki HA, Schiffman M, Castle PE, Fetterman B, Poitras NE, Lorey T, Cheung LC, Raine-Bennett T, Gage JC, Kinney WK (2013) Five-year risk of CIN3+ and cervical cancer for women who test Papnegative butare HPV-positive.J Low Genit TractDis 17(501):56-63

14. Kelly R, Walker P, Kitchener H, Moss S (2012) Incidence of cervical intraepithelial neoplasia grade 2 or worse in colposcopy-negative/human papillomavirus-positive women with low-grade cytological abnormalities. BJOG 119:20-25

15. Kühn W (2011) Kolposkopie zur Früherkennung des Zervixkarzinoms. Pathologe 32:497-504

16. Kühn W, Gieseking F (2015) Die aktuellen Empfehlungen der AG CPC zur Kolposkopie. gyn 20:25-47

17. Kyrgiou M, Kalliala IEJ, Mitra A, Fotopoulou C, Ghaem-Maghami S, Martin-Hirsch PPL, CruickshankM, Arbyn M, ParaskevaidisE (2017)Immediate referral to colposcopy versus cytological surveillance for minor cervical cytological abnormalities in the absence of HPV test. Cochrane Database Syst Rev 1:CD9836. https://doi.org/10.1002/14651858. CD009836.pub2

18. Lee DJ, Oh Y-h LSO, Kim JY (2012) Comparison of diagnostic cytomorphology of atypical squamous cells in liquid-based preparations and conventional smears. Korean J Pathol 46:365-369

19. Marquardt K, Stubbe M, Broschewitz U (2016) Zervixkarzinome in Mecklenburg-Vorpommern. Pathologe 37:78-83

20. Marquardt K, Ziemke P (2018) Münchner Nomenklatur III: Klassifikation nach Risiko. Pathologe 39(1):57-64. https://doi.org/10.1007/s00292017-0382-x

21. Marquardt K, Kossowski I, Hantschke-Zerbich $H_{\text {, }}$ MichelM (2019) An der Schwellezumorganisierten Zervixkarzinomscreening. Befunde der Münchner Nomenklatur III und zytologisch-histologische Korrelation.Gynäkologe. https://doi.org/10.1007/ s00129-019-4417-7 
22. Martin-Hirsch PPL, Paraskevaidis E, Bryant A, Dickinson HO, Keep SL (2013) Surgery for cervical intraepithelial neoplasia. Cochrane Database Syst Rev. https://doi.org/10.1002/14651858. CD001318.pub2

23. Massad LS, Einstein MH, Huh WK, Katki HA, Kinney WK, Schiffman M, Solomon D, Wentzensen N, Lawson HW, 2012 ASSCP Consensus Guidelines Conference (2013) 2012 updated consensus guidelines for the management of abnormal cervical cancer screening tests and cancer precursors. J Low Genit Tract Dis 17(5 Suppl 1):1-27

24. Mayeaux EJ, Novetsky AP, Chelmov D, Garcia F, Choma K, Liu AH, Papasozomenos T, Einstein MH, Massad LS, Wentzensen N, Waxman AG, Conageski C, Khan MJ, Huh WK (2017) ASCCP Colposcopy standards: Colposcopy quality improvement recommendations for the United States. J Low Genit Tract Dis 21:242-248

25. Nayar R, Wilbur DC (2015) The Bethesda system for reporting cervical cytology, 3. Aufl. Springer, New York

26. Robert Koch-Institut, Gesellschaft der epidemiologischen Krebsregister in Deutschland e. V. (Hrsg) (2017) Krebs in Deutschland 2013/2014. Robert Koch-Institut und die Gesellschaft der epidemiologischen Krebsregister in Deutschland e. V., Berlin https://doi.org/10.17886/rkipubl-2017-007

27. Stoler M, Bergeron C, Colgan TC, Ferenczy AS, Herrington CS, Kim K-R, Loening T et al (2014) Squamous cell tumours and precursors. In: Kurman RJ, Carcanglu ML, Herrington CS, Young RH (Hrsg) WHO classification of tumours of female reproductive organs, 4. Aufl. WHO Press, Lyon, S 172-176

28. Wang J, Andrae B, Sundström K, Ström P, Ploner A, Elfström KM, Arnheim-Dahlström L, Dillner J, Sparén P (2016) Risk of invasive cervical cancer after atypical glandular cells in cervical screening: nationwide cohort study. BMJ 352:i276. https:// doi.org/10.1136/bmj.i276

29. Zheng B, Yang H, Li Z, Wei G, You J, Liang X, Zhao C (2017) HPV test results and histological followup results of patients with LSIL Cervical Cytology from the Largest CAP-certified laboratory in China. JCancer 8(13):2436-2441

\section{Rheuma häufig Nebenwirkung von neuer Krebsbehandlung}

Checkpoint-Inhibitoren und Autoimmunreaktionen

Sogenannte Checkpoint-Inhibitoren können Krebserkrankungen heilen, indem sie die körpereigene Immunabwehr verstärken. Diese Medikamente aktivieren dabei jedoch körpereigene T-Zellen, dieselben Zellen, die an der Entstehung der rheumatoiden Arthritis (RA) und anderer Autoimmunerkrankungen beteiligt sind. Gelenkbeschwerden gehören deshalb zu häufigen Nebenwirkungen der Therapie mit Checkpoint-Inhibitoren.

Seit 2011 wurden in Deutschland sechs

Checkpoint-Inhibitoren zugelassen. Die Antikörper Ipilimumab, Nivolumab, Pembrolizumab, Atezolizumab, Durvalumab und Avelumab verhindern auf unterschiedliche Weise, dass Krebszellen sich der Abwehr durch T-Zellen entziehen können. „Die Angriffsbereitschaft der T-Zellen wird gesteigert und vormals unheilbare Krebserkrankungen wie das Melanom und Lungenkrebs drängt das Immunsystem des Körpers zurück", erläutert Professor Dr. med Hendrik SchulzeKoops, Präsident der DGRh und Leiter der Rheumaeinheit des Klinikums der LudwigMaximilians-Universität München.

\section{Häufig „Autoimmunphänomene" während der Therapie}

Die Angriffslust der T-Zellen bleibt jedoch nicht auf die Tumoren beschränkt. Sie können auch gesunde körpereigene Zellen angreifen und sind wichtiger Akteur bei Autoimmunerkrankungen wie der RA. „Folge ist, dass es während der Behandlung mit Checkpoint-Inhibitoren häufig zu Autoimmunphänomenen kommt", sagt Professor Schulze-Koops. Bis zu 70 Prozent der Patienten erleiden während einer Therapie beispielweise Muskel- oder Gelenkschmerzen oder auch eine Entzündung der Tränen- oder Speicheldrüsen, wodurch es zu einer Trockenheit der Schleimhäute kommt. In Einzelfällen werden auch die Blutgefäße angegriffen oder es kommt zu Autoimmunerkrankungen von Drüsen, des Darmes, der Haut oder von anderen inneren Organen. Männer sind dabei ebenso häufig betroffen wie Frauen.

Behandlung mit bekannten RheumaMedikamenten

Da die Antikrebswirkung der CheckpointInhibitoren von der Aktivierung der T-Zellen abhängt, sind auch die Immunnebenwirkungen umso stärker, je besser die Medikamente wirken. Professor Schulze-Koops sagt: „Etwa zwei Drittel der Patienten, bei denen sich der Tumor teilweise oder ganz zurückbildet, leiden unter den Immunnebenwirkungen." Starke Gelenkbeschwerden oder andere Autoimmunphänomenen seien deshalb im Prinzip ein gutes Zeichen für die Patienten, so der Experte "Wir wissen inzwischen auch, wie wir ihnen helfen können, ohne zu schaden", so der Rheumatologe. Die Patienten würden heute mit den gleichen Medikamenten behandelt, die auch bei Rheuma-Erkrankungen zum Einsatz kommen. Schwere Schübe werden mit Kortison abgefangen, danach erhalten die Patienten Methotrexat, das seit langem ein Standardmedikament in der Behandlung von rheumatischen Erkrankungen ist. „Entscheidend ist, dass im Rahmen einer Therapie mit Checkpoint-Inhibitoren rechtzeitig ein Rheumatologe hinzugezogen wird, sobald es zu entsprechenden Symptomen kommt", so der Präsident der DGRh. Umgehend behandelt, können Langzeitfolgen der modernen Krebstherapie so gut verhindert werden. Dafür arbeiten Krebsspezialisten und Rheumatologen in der Therapie dieser Patienten eng zusammen.

\section{Janina Wetzstein, Deutsche Gesellschaft für Rheumatologie}


Hier steht eine Anzeige.

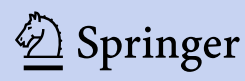

